\title{
RESPUESTA INELÁSTICA DE MARCOS DE ACERO CON INTERACCIÓN INERCIAL SUELO - ESTRUCTURA
}

\author{
Luciano Roberto Fernández Sola ${ }^{(1)}$, Edgar Tapia Hernández ${ }^{(1)}$ y David Dávalos Chávez ${ }^{(2)}$
}

\begin{abstract}
RESUMEN
En este artículo se discuten los resultados de análisis no lineales ante carga estática monótona creciente de marcos de acero de edificios regulares para evaluar la influencia de la interacción dinámica suelo - estructura (IDSE) en su respuesta inelástica. Los marcos, que forman parte de edificios regulares de 8, 12 y 16 pisos, fueron diseñados para la condición de suelo blando de la Ciudad de México y se estudian a partir de distintas proporciones de rigidez lateral (marcos con dos crujías contraventeadas, una crujía contraventeada y sin contravientos) y de la flexibilidad de la cimentación (cimentación rígida, pilotes y cajón de cimentación). La flexibilidad del sistema suelo cimentación se modeló mediante un conjunto de resortes en la base cuyas constantes equivalen a la magnitud de las funciones de impedancia para las frecuencias fundamentales de cada edificio. El artículo compara la ductilidad que desarrollan los modelos con base flexible y con base rígida y las diferencias entre estas ductilidades con las que se calculan mediante las metodologías propuestas en los reglamentos nacionales especializados.
\end{abstract}

Palabras clave: marcos de acero; ductilidad; sobrerresistencia; desempeño estructural

\section{INELASTIC RESPONSE OF STEEL FRAMES WITH INERTIAL SOIL STRUCTURE INTERACTION}

\begin{abstract}
In this paper, results of nonlinear pushover analyses of steel frames of regular buildings are discussed in order to evaluate the influence of Dynamic Soil - Structure Interaction in their inelastic response. The studied frames are part of regular steel buildings of 8-, 12- and 16-stories, which were designed for soft soil conditions of Mexico City. Different lateral stiffness (two braced bays, one braced bay and no braces) and foundation's flexibility (rigid base, piles and mat foundation) were studied. Soilfoundation flexibility is introduced by a set of springs in the base, where their constants are equivalent to the values of the impedance functions at fundamental frequencies of each building. The paper compares the ductility developed by the frames with flexible base against the developed by the frames with rigid base and the proposed methodology of specialized codes.
\end{abstract}

Keywords: steel frames; ductility; overstrength; structural performance

\footnotetext{
Artículo recibido el 22 de septiembre de 2014 y aprobado para su publicación el 30 de julio de 2015. Se aceptarán comentarios y/o discusiones hasta cinco meses después de su publicación.

(1) Profesor, Departamento de Materiales, Universidad Autónoma Metropolitana Azcapotzalco, Av. San Pablo 180, Col. Reynosa Tamaulipas,02200 México, DF,lrfs@azc.uam.mx,etapiah@azc.uam.mx

(2) Estudiante de Maestría, Posgrado en Ingeniería Estructural, Universidad Autónoma Metropolitana - Azc. Actualmente, Alonso y Asociados, Proyecto Estructural, Av. De la Palma 150-101, México, DF, david@alonsoasociados.com.mx
} 


\section{INTRODUCCIÓN}

La tendencia de diseño sismorresistente de la mayoría de los reglamentos internacionales permite que las estructuras ingresen en el intervalo inelástico durante sismos intensos, lo que proporciona dos ventajas fundamentales: un mecanismo de disipación de energía y una reducción significativa de la resistencia necesaria. Esta capacidad de las estructuras de ingresar en el intervalo de comportamiento plástico de manera estable se sustenta en la respuesta que los elementos estructurales individualmente son capaces de desarrollar, pero también en la interacción del sistema como un conjunto (incluyendo la cimentación).

En general, el estudio de la respuesta dinámica de las estructuras es un fenómeno complejo que depende de muchas variables: el comportamiento individual de los elementos que conforman la estructura, la respuesta inelástica global de la estructura considerando las posibles redistribuciones de carga en el intervalo inelástico, las posibles fuentes de excitación y sus características particulares, la respuesta dinámica del suelo y su interacción con la cimentación, etc. En este sentido, es común que el ingeniero tome decisiones durante el proceso de diseño que pretenden simplificar la complejidad y reducir el tiempo de ejecución y post-proceso de los análisis.

Entre ellas, una de las decisiones más recurrentes consiste en suponer que el sistema suelocimentación es lo suficientemente rígido como para despreciar sus efectos en los análisis. Sin embargo, esa simplificación no es correcta en todos los casos, puesto que en menor o mayor magnitud la base de la edificación tiene desplazamientos relativos durante un sismo intenso. Así, una condición flexible entre el sistema suelo - cimentación - estructura modificaría las propiedades dinámicas del sistema, lo que podría implicar una redistribución de cargas imprevista entre los elementos sismo-resistentes. Esa variación de la respuesta dinámica del suelo y de la estructura provocadas por la flexibilidad del sistema suelo cimentación en un movimiento sísmico es conocida como interacción dinámica suelo - estructura (IDSE).

La mayoría de los reglamentos especializados consideran los efectos de la IDSE, a partir de una proporción que se establece en función de criterios para calcular el periodo de la estructura y amortiguamiento modificados por la flexibilidad de la base. Sin embargo, otras variables de la respuesta de la estructura, como el efecto $P-\Delta$ en las columnas, puede incrementarse con el aumento de flexibilidad de la cimentación, especialmente en edificios flexibles. De hecho, algunos estudios como Halabian y Kabiri (2004) y Barcenas y Esteva (2006) han demostrado variaciones importantes en los desplazamientos máximos que desarrollan los modelos de estructuras de concreto armado cuando se incluye la IDSE, así como variaciones en las demandas en los elementos estructurales.

Así, en esta investigación se evalúa la influencia de la interacción dinámica suelo - estructura en la respuesta inelástica en marcos de acero, que forman parte de edificios regulares de 8,12 y 16 pisos, que fueron diseñados para las mayores demandas de aceleración propuestas para la Ciudad de México. Adicionalmente, en el estudio se ha incluido como variable:

a. La rigidez lateral de los modelos al considerar marcos con dos crujías contraventeadas, marcos con una crujía contraventeada y marcos sin contravientos.

b. La flexibilidad del sistema suelo-cimentación mediante la consideración de modelos con cimentación rígida, cimentación con pilotes y modelos desplantados en un cajón de cimentación. 


\section{CRITERIOS NORMATIVOS}

Las Normas Técnicas Complementarias para el Diseño por Sismo (NTCS-04) del Reglamento de Construcciones para el Distrito Federal (RCDF-04) y el Manual de Obras Civiles de la Comisión Federal de Electricidad (MOCS-08) proponen un procedimiento para incorporar los efectos de la IDSE mediante una modificación del periodo de la estructura y su amortiguamiento; de manera que la flexibilización del sistema conlleva implícitamente la posibilidad de que la demanda de aceleración espectral sea modificada. Esta metodología establece que una vez que se determine el cortante basal correspondiente a la aceleración espectral para el periodo y el amortiguamiento modificados, el resto de las respuestas se modifiquen en la misma proporción.

En los reglamentos internacionales vigentes, las modificaciones asociadas con la IDSE más frecuentemente consideradas son: a) el alargamiento del periodo estructural y b) la modificación del amortiguamiento, debido a que son las más conocidas (Wolf 1985). Pese a esto, otras variables también deberían ser modificadas, sobre todo las que pretenden proporcionar al diseñador una estimación realista de la respuesta inelástica; por ejemplo, la ductilidad $\mu$ que desarrollará la estructura.

Específicamente, las NTCS-04 y el MOCS-08 establecen un procedimiento para determinar la modificación de la ductilidad debida a la flexibilización provocada por los efectos de la IDSE. Esta modificación, propuesta por Rosenbleth y Resendiz (1988) y reformulada posteriormente por Pérez-Rocha y Avilés (2003), se basa en que la IDSE varía la rigidez inicial $K_{\text {elast }}$ (figura 1 ) debido a la flexibilidad del sistema suelo - cimentación; esto ocasiona que la deformación de fluencia $U_{y}$ tenga una magnitud más grande de la que se obtendría del análisis con base rígida y, por consiguiente, también se modifica la deformación última $U_{u}$. Entonces, si las deformaciones que definen la ductilidad $\mu$ se alteran, la ductilidad que puede desarrollar el sistema se reduce (por el incremento de $U_{y}$ ), pese a que la estructura tenga la misma capacidad de deformación inelástica.

En ese sentido, la deformación de los análisis con base rígida están asociados únicamente a la deformación de la estructura $U$ (figura $1 \mathrm{~b}$ ); mientras que el desplazamiento de los análisis con base flexible depende del deformación de la estructura $U$ y de un desplazamiento adicional provocado por la flexibilidad de la cimentación $U_{c}$ que resulta de la suma del desplazamiento del giro del sistema $\phi h$ más el movimiento de traslación de la cimentación $U_{C I M E N T}$.

a)

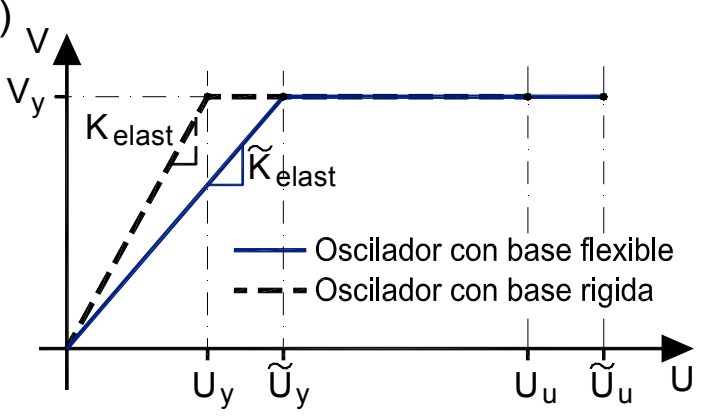

a) Relaciones fuerza-deformación idealizadas b)

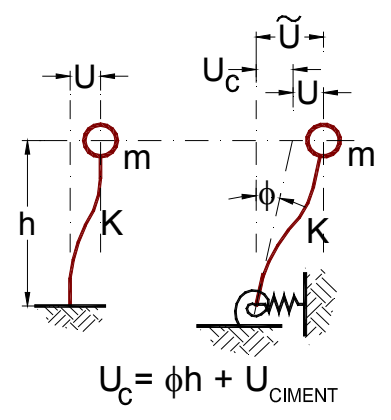

b) Definición de deformaciones

Figura 1. Influencia de la base flexible en un oscilador (adaptada de Pérez-Rocha y Avilés 2003) 
De esta manera, la reducción teórica de la ductilidad puede determinarse con la ecuación 1, que está incluida en el Apéndice A de las NTCS-04, la cual es función de la relación entre el periodo de la estructura sobre base rígida $T_{e}$ y el periodo considerando la base flexible $\tilde{T}_{e}$.

$\tilde{\mu}=\frac{T_{e}^{2}}{\tilde{T}_{e}^{2}}(\mu-1)+1$

La ecuación anterior se desarrolló a partir de estudios del comportamiento de un oscilador de un grado de libertad (Rosenbleth y Resendiz 1988; Pérez-Rocha y Avilés 2003), que es una simplificación usada en investigaciones similares donde comúnmente la estructura se modela con un grado de libertad y el sistema de suelo - cimentación se modela con dos o tres grados de libertad (por ejemplo Ghannad y Jahankhah 2007 y Mashuli y Ghannad 2009). En contraparte, otros estudios como Ganjavi y Hao (2011) han demostrado que las demandas de resistencia y ductilidad en análisis de estructuras con múltiples grados de libertad pueden ser muy diferentes de las que se obtienen de análisis con un grado de libertad equivalente, dependiendo del número de pisos, la flexibilidad del suelo y el grado de inelasticidad. Por lo anterior, en esta investigación se pretende ampliar el conocimiento sobre el comportamiento inelástico de estructuras flexibles con múltiples grados de libertad con distintas condiciones de cimentación y evaluar los criterios de predicción de los reglamentos vigentes.

\section{DESCRIPCIÓN DE LOS MODELOS}

Los edificios estudiados se diseñaron siguiendo estrictamente los requisitos propuestos en el RCDF04 para que los marcos de acero (trabes y columnas) en cada entrepiso sean capaces de resistir, sin considerar la contribución del sistema de contravientos, cuando menos el 50 por ciento de la fuerza sísmica actuante. En Tapia y Tena (2011) y Dávalos (2013) es posible encontrar más información de los modelos diseñados.

En la figura 2 se muestran las dimensiones en planta y elevación de los tres tipos de marcos considerados en este estudio: marco central sin contravientos, marco intermedio con una crujía contraventeada y marco perimetral con dos crujías contraventeadas.

a)

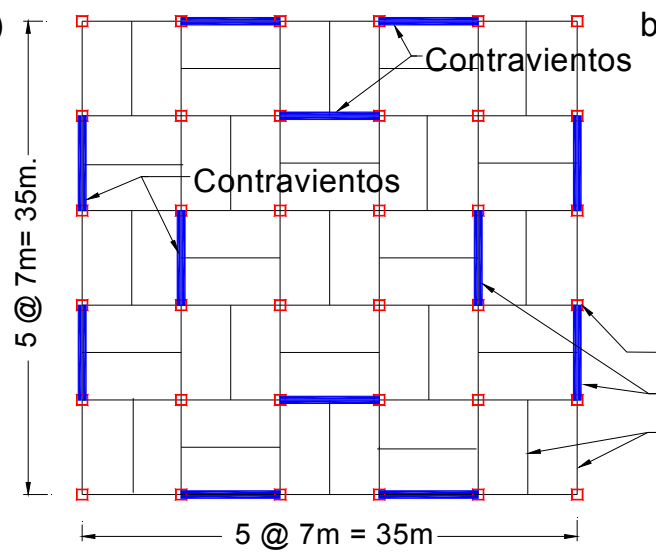

b)

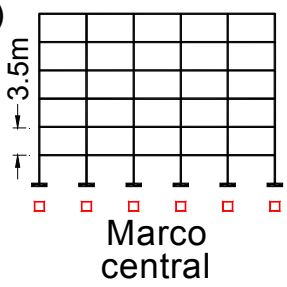

Columnas (Sección rectangular A50)

Contravientos (Sección rectangular A36)

Vigas (Sección I A36)

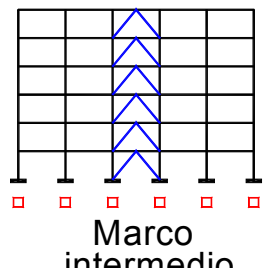

intermedio

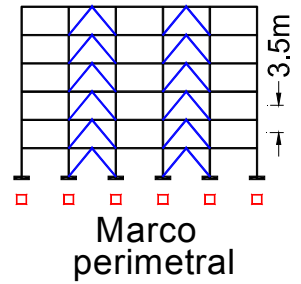

perimetral

Figura 2. a) Planta tipo de los edificios, b) elevación de los marcos estudiados

Los modelos de los edificios se supusieron localizados en la zona IIIb de la subzonificación sísmica del Distrito Federal (NTCS-04) para asociarlos a las mayores demandas de aceleración propuestas en el 
reglamento para suelo blando con poca rigidez rotacional. Las secciones transversales finales de los análisis que satisfacen estrictamente las restricciones de resistencia y rigidez del RCDF-04 se muestran en la tabla 1; mientras que, en la tabla 2 se presenta la información dinámica de los análisis elásticos realizados.

Adicionalmente, se realizaron análisis no lineales ante carga estática monótona creciente en el programa Drain2DX (Prakash et al., 1992) para verificar que la respuesta inelástica fuera congruente con las premisas de diseño: mecanismo de colapso columna fuerte - viga débil- contraviento más débil con una adecuada distribución en la altura de la respuesta inelástica. Para ejemplificar esto, en la figura 3 se muestran las instantáneas de fluencia normalizadas por intervalos en código de colores de la secuencia de formación del mecanismo de colapso del marco intermedio y perimetral del modelo de 8 niveles. En la figura 3 también se reporta la distorsión global última $\left(\delta_{\text {global }}\right)$, definida como la deformación lateral en la azotea dividida entre la altura total del edificio. En Tapia y Tena (2008) es posible conocer otros aspectos de la respuesta elástica e inelástica de los edificios estudiados.

Tabla 1. Perfiles estructurales de los modelos estudiados

\begin{tabular}{|c|c|c|}
\hline Modelo & Identificación del elemento & Sección transversal $(\mathrm{cm})$ \\
\hline \multirow{6}{*}{$\begin{array}{l}\text { Edificio de } \\
8 \text { pisos }\end{array}$} & Columnas, niveles N1 - N3 & Cajón 40x40 e= $1.91 \mathrm{~cm}(3 / 4 ”)$ \\
\hline & Columnas, niveles N4 - N6 & Cajón $40 \times 40 \mathrm{e}=1.58 \mathrm{~cm}\left(5 / 8^{\prime \prime}\right)$ \\
\hline & Columnas, niveles N7 - N8 & Cajón $40 \mathrm{x} 40 \mathrm{e}=1.27 \mathrm{~cm}(1 / 2 ")$ \\
\hline & Trabes, todos los niveles & IR $45.7 \times 112.9 \mathrm{kgf} / \mathrm{m}$ \\
\hline & Contravientos, niveles N1 - N4 & Cajón $15 \times 15 \mathrm{e}=0.95 \mathrm{~cm}(3 / 8 ”)$ \\
\hline & Contravientos, niveles N5 - N8 & Cajón $15 \times 15 \mathrm{e}=0.64 \mathrm{~cm}(1 / 4 ”)$ \\
\hline \multirow{8}{*}{$\begin{array}{l}\text { Edificio de } \\
12 \text { pisos }\end{array}$} & Columnas, niveles N1 - N3 & Cajón $45 \times 45 \mathrm{e}=2.22 \mathrm{~cm}\left(7 / 8^{\prime \prime}\right)$ \\
\hline & Columnas, niveles N4 - N6 & Cajón $45 \times 45 \mathrm{e}=1.90 \mathrm{~cm}\left(3 / 4^{\prime \prime}\right)$ \\
\hline & Columnas, niveles N7 - N8 & Cajón $45 \times 45 \mathrm{e}=1.58 \mathrm{~cm}\left(5 / 8^{\prime \prime}\right)$ \\
\hline & Columnas, niveles N9 - N12 & Cajón $45 \times 45 \mathrm{e}=1.27 \mathrm{~cm}(1 / 2 ”)$ \\
\hline & Trabes, todos los niveles & IR $45.7 \times 144.3 \mathrm{kgf} / \mathrm{m}$ \\
\hline & Contravientos, niveles N1 - N4 & Cajón $25 \times 25 \mathrm{e}=2.22 \mathrm{~cm}\left(7 / 8^{\prime \prime}\right)$ \\
\hline & Contravientos, niveles $\mathrm{N} 5-\mathrm{N} 8$ & Cajón $25 \times 25 \mathrm{e}=1.90 \mathrm{~cm}(3 / 4 ")$ \\
\hline & Contravientos, niveles N9 - N12 & Cajón $25 \times 25$ e=1.58 cm (5/8”) \\
\hline \multirow{8}{*}{$\begin{array}{l}\text { Edificio de } \\
16 \text { pisos }\end{array}$} & Columnas, niveles N1 - N4 & Cajón $50 \times 50 \mathrm{e}=2.54 \mathrm{~cm}\left(17 / 8^{\prime \prime}\right)$ \\
\hline & Columnas, niveles N5 - N8 & Cajón $50 \times 50 \mathrm{e}=1.91 \mathrm{~cm}(13 / 4 ")$ \\
\hline & Columnas, niveles N9 - N12 & Cajón $50 \times 50 \mathrm{e}=1.58 \mathrm{~cm}(1 \mathrm{1} / 2 ")$ \\
\hline & Columnas, niveles N13 - N16 & Cajón $50 \times 50 \mathrm{e}=1.27 \mathrm{~cm}(13 / 8 ")$ \\
\hline & Trabes, todos los niveles & IR $55.3 \times 150.9 \mathrm{~kg} / \mathrm{m}$ \\
\hline & Contravientos, niveles N1 - N6 & Cajón $35 \times 35 \mathrm{e}=3.49 \mathrm{~cm}\left(1 \mathrm{3} / 8^{\prime \prime}\right)$ \\
\hline & Contravientos, niveles N7 - N11 & Cajón $35 \times 35 \mathrm{e}=3.18 \mathrm{~cm}(1 \mathrm{1} / 4 ")$ \\
\hline & Contravientos, niveles N12 - N16 & Cajón $35 \times 35 \mathrm{e}=2.86 \mathrm{~cm}\left(11 / 8^{\prime \prime}\right)$ \\
\hline
\end{tabular}

Es valioso hacer notar que la respuesta inelástica de los edificios tiene una dependencia de la altura de los modelos, pese a que fueron diseñados con la misma filosofía y condiciones de diseño (Tapia y Tena 2008). Esto es, los modelos más altos son menos dúctiles y desarrollan un mecanismo de colapso con una menor distribución de la respuesta inelástica en la altura. Con el propósito de hacer notar esta tendencia, en la tabla 2 se muestra la ductilidad global obtenida de la curva de capacidad (cortante basal contra distorsión en la azotea) y la ductilidad global teórica que se obtuvo de curvas bilineales idealizadas. Es posible consultar otros detalles sobre la definición de estas curvas y una amplia discusión sobre la dependencia de la altura de los edificios y la ductilidad que pueden desarrollar en Tapia y Tena (2011). 
Tabla 2. Información dinámica de los edificios desplantados sobre base rígida

\begin{tabular}{|c|c|c|c|c|c|}
\hline \multirow[b]{2}{*}{ Modelo } & \multirow{2}{*}{$\begin{array}{l}\text { Altura del } \\
\text { edificio, } H_{T} \\
\quad(\mathrm{~m})\end{array}$} & \multirow{2}{*}{$\begin{array}{l}\text { Periodo } \\
\text { fundamental } \\
\text { (s) }\end{array}$} & \multirow{2}{*}{$\begin{array}{c}\text { Frecuencia } \\
\text { circular } \\
(\mathrm{rad} / \mathrm{s})\end{array}$} & \multicolumn{2}{|c|}{ Ductilidad global $\mu$} \\
\hline & & & & $\begin{array}{l}\text { Curva de } \\
\text { capacidad }\end{array}$ & $\begin{array}{c}\text { Curva bilineal } \\
\text { idealizada }\end{array}$ \\
\hline Edificio de 8 pisos & 28.0 & 0.40 & 15.71 & 2.64 & 1.97 \\
\hline Edificio de 12 pisos & 42.0 & 0.50 & 12.57 & 2.10 & 1.89 \\
\hline Edificio de 16 pisos & 56.0 & 0.63 & 9.97 & 1.99 & 1.86 \\
\hline
\end{tabular}

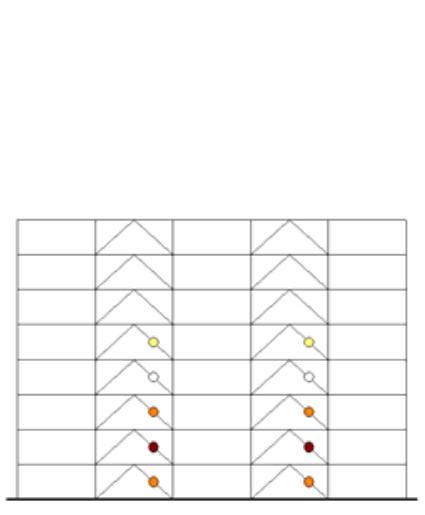

Paso $25\left(\delta_{\text {global }}=0.284 \%\right)$

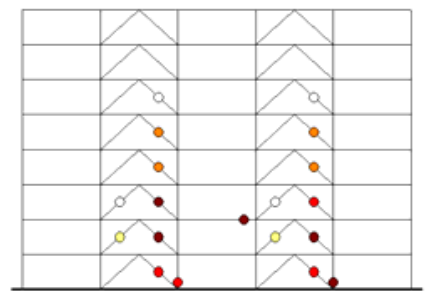

Paso $31\left(\delta_{\text {global }}=0.218 \%\right)$
$0.00-0.20 \bigcirc$

$0.20-0.40 \bigcirc$

$0.40-0.60$

$0.60-0.80$

$0.80-1.00$

Marco perimetral con dos crujías contraventeadas

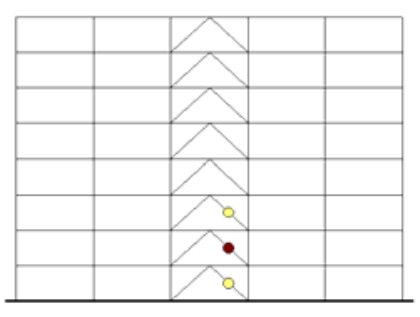

Paso $14\left(\delta_{\text {global }}=0.155 \%\right)$

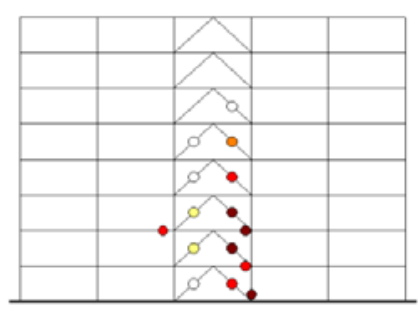

Paso $21\left(\delta_{\text {global }}=0.218 \%\right)$

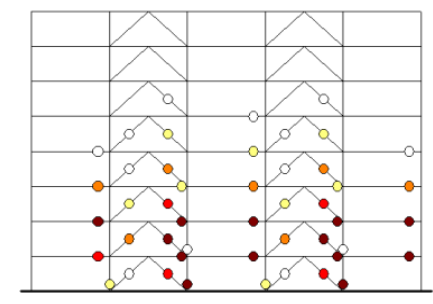

Paso $34\left(\delta_{\text {global }}=0.648 \%\right)$

Marco intermedio con una crujía contraventeada

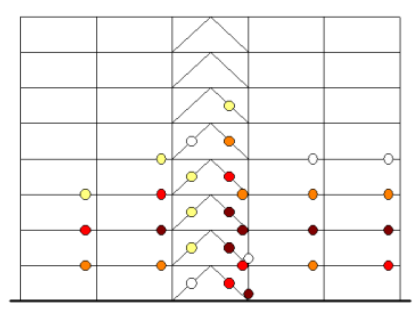

Paso $26\left(\delta_{\text {global }}=0.431 \%\right)$

Figura 3. Distribución de articulaciones plásticas en el colapso del modelo de 8 pisos con base rígida

En esta investigación, el desplazamiento de fluencia $U_{y}$ se define como el punto de intersección entre la recta con pendiente igual a la rigidez elástica del sistema $K_{\text {elast }}$ y la línea horizontal que define el cortante último. Es decir, los resultados que se discutirán a continuación están basados en la curva bilineal idealizada que se obtiene a partir de la curva de capacidad. Esta decisión pretende ajustar los resultados con el modelo elasto-plástico perfecto utilizado por Pérez-Rocha y Avilés (2003) para evaluar el criterio de las NTCS-04.

\section{Características del suelo de desplante}

En consistencia con las suposiciones de diseño, los perfiles estratigráficos para modelar la respuesta del suelo se obtuvieron con las propiedades reportadas en un Estudio de Mecánica de Suelos (ESVM 1986) de la Colonia Roma Sur, Delegación Cuauhtémoc (coordenadas: $19^{\circ} 24 \mathrm{~N}$ y $99^{\circ} 10 \mathrm{~W}$ ) de la zona $I I I b$. El estudio forma parte de un proyecto llamado Estudios sobre sismicidad en el Valle de México, donde se realizaron pruebas in situ para obtener las velocidades de propagación de ondas de cortante por dos métodos de campo: pozo abajo (Downhole) y por sonda suspendida. 
Las propiedades del suelo se definieron con base en el perfil de velocidades de propagación de ondas mostrado en la figura 4 correspondiente a la Zona del Lago. Como se aprecia, las propiedades del suelo (tabla 3) no presentan variaciones importantes hasta los 40 metros de profundidad, por lo que en este estudio, el suelo se idealizó como un sólo estrato.

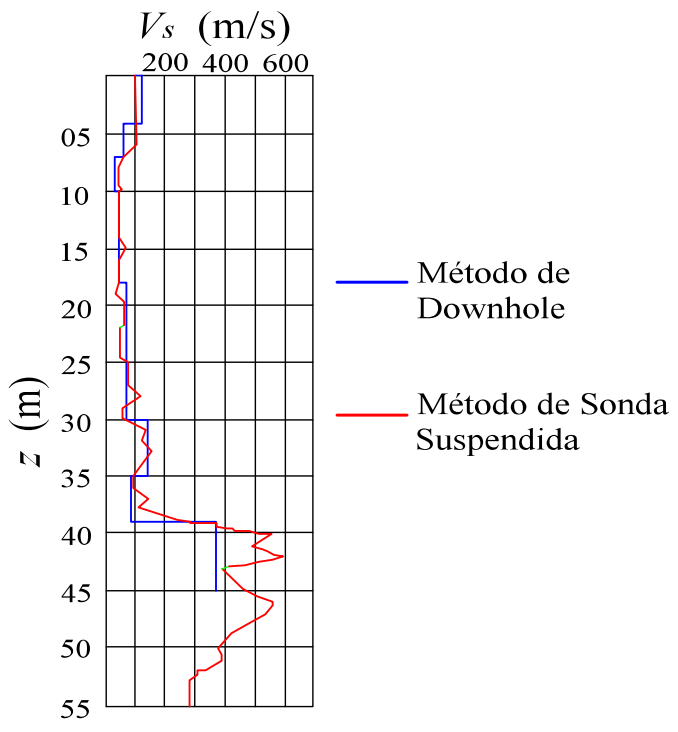

Figura 4. Variación de la velocidad de propagación de ondas de cortante (adaptada de ESVM 1988)

Tabla 3. Propiedades mecánicas del suelo

\begin{tabular}{|c|c|c|}
\hline \multirow[b]{2}{*}{ Propiedad } & \multicolumn{2}{|c|}{ Magnitud } \\
\hline & $\begin{array}{c}\text { Estudio de } \\
\text { Mecánica de } \\
\text { Suelos de } 1988\end{array}$ & $\begin{array}{c}\text { Proyección al } \\
2013\end{array}$ \\
\hline Periodo del suelo estimado (Ts) & $2.34 \mathrm{~s}$ & $1.85 \mathrm{~s}$ \\
\hline Cohesión, $c$ & \multicolumn{2}{|c|}{$5.0 \mathrm{t} / \mathrm{m}^{2}$} \\
\hline Velocidad de propagación de ondas de corte, $V_{s}$ & $65.0 \mathrm{~m} / \mathrm{s}$ & $77.9 \mathrm{~m} / \mathrm{s}$ \\
\hline Peso volumétrico, $\gamma$ & \multicolumn{2}{|c|}{$1.30 \mathrm{t} / \mathrm{m}^{3}$} \\
\hline Módulo de Poisson, $v$ & \multicolumn{2}{|c|}{0.49} \\
\hline Amortiguamiento, $\xi$ & \multicolumn{2}{|c|}{0.05} \\
\hline
\end{tabular}

Ahora, es conocido que el bombeo de agua en la Ciudad de México ha generado una pérdida en la presión de agua del subsuelo, induciendo a una consolidación acelerada. Esto ha provocado una reducción gradual del espesor de los depósitos y, por consiguiente, alteraciones en las propiedades dinámicas del suelo. Por esta razón, fue necesario adecuar las propiedades del suelo obtenidas en ESVM (1988) por causa del hundimiento regional del subsuelo del Valle de México con el propósito de obtener propiedades aproximadas modificadas para las condiciones cuando se desarrolló esta investigación (2013). Esta adecuación se realizó mediante el procedimiento propuesto por Aguilar et al. (2003). 
Aguilar et al. (2003) estudiaron la evolución del hundimiento regional y su relación con la modificación de las propiedades del suelo, a partir de datos periódicos de bancos de nivel en la Zona del Lago y la Zona de Transición entre 1983 y 1998. Con esta información, relacionaron la velocidad de hundimiento con el espesor del suelo compresible, lo que permitió estimar la variación de esa velocidad en función del tiempo. Asimismo, Aguilar et al. (2003) establecieron el cambio del periodo fundamental del suelo $T_{s}$ debido a este fenómeno, a partir de la relación entre la velocidad de hundimiento, espesor comprensible de los estratos $H_{s}$ y la velocidad de propagación de las ondas de corte $V_{s}$.

De esta manera, el periodo dominante de vibración del suelo inicial se estima mediante la ecuación 2. Luego, a partir de gráficas que son función del tiempo transcurrido, es posible estimar el periodo dominante del suelo en el tiempo actual $T_{s 2013}$ (en este caso al 2013), que se utiliza para obtener la velocidad de propagación al 2013 mediante la ecuación 3 (Aguilar et al., 2003).

$$
\begin{aligned}
T_{s_{1988}} & =\frac{4 H_{s_{1988}}}{V_{s_{1988}}} \\
V_{s_{2013}} & =\frac{124\left(T_{s_{2013}}-0.5\right)^{1 / 2}}{T_{s_{2013}}}
\end{aligned}
$$

En las ecuaciones anteriores, $H_{S} 1988$ es el espesor del estrato cuando se realizó el Estudio de Mecánica de Suelos que se consideró igual a $38 \mathrm{~m}$ (figura 4); $V_{s} 1988$ es la velocidad del estrato igual a 65 m/s (tabla 3) ESVM (1988); $T_{s} 2013$ es el periodo dominante del suelo que se estimó mediante el procedimiento descrito en Aguilar et al. (2003) considerando que habían transcurrido 25 años y $V_{s 2013}$ es la velocidad estimada al 2013 (tabla 3).

Como consecuencia del hundimiento regional (cuya proyección para el sitio de estudio es de $2 \mathrm{~m}$ en el intervalo de tiempo considerado) es natural que la cohesión $c$ y el peso volumétrico del suelo $\gamma$ también se hayan modificado; sin embargo, la estimación realizada se concentra únicamente en las propiedades dinámicas del suelo que tienen influencia en la magnitud de las funciones de impedancia, por lo que con fines prácticos se aceptaron las magnitudes publicadas en ESVM (1988). Se hace notar que el procedimiento sugiere un incremento de la velocidad de propagación de ondas de cortante de casi el 20 por ciento en 25 años (tabla 3).

Con esta información, se calcularon dos propuestas de cimentación de los edificios independientes entre sí para considerar los efectos de flexibilidad: a) un cajón de cimentación y b) pilotes de fricción. El pre-diseño por resistencia se realizó siguiendo los lineamientos de las Normas Técnicas Complementarias para el Diseño de Cimentaciones (NTCC-04).

El cajón se diseñó como una cimentación parcialmente compensada, donde la compensación corresponde a la diferencia entre la capacidad de carga del cajón como una losa y el peso total de la edificación. Los pilotes se diseñaron primordialmente a fricción y se ajustaron sus dimensiones para determinar números de pilotes que pudieran ser distribuidos en un arreglo simétrico. En ambos casos, el pre-diseño fue regido por la combinación de cargas verticales. En la tabla 4 se muestran las principales características de las propuestas de los tres edificios considerados en esta investigación. Es posible conocer otros detalles del procedimiento de diseño de los sistemas de cimentación en Dávalos (2013). 
Tabla 4. Características geométricas de las cimentaciones

\begin{tabular}{cccc}
\hline \multirow{2}{*}{ Edificio } & Cajón de cimentación & \multicolumn{2}{c}{ Pilotes de fricción } \\
\cline { 2 - 4 } & $\begin{array}{c}\text { Profundidad de } \\
\text { desplante, } D\end{array}$ & $\begin{array}{c}\text { Cantidad de } \\
\text { pilotes }\end{array}$ & $\begin{array}{c}\text { Longitud del } \\
\text { pilote }\end{array}$ \\
\hline 8 niveles & $4.00 \mathrm{~m}$ & 121 & $20.00 \mathrm{~m}$ \\
\hline 12 niveles & $9.00 \mathrm{~m}$ & 169 & $22.50 \mathrm{~m}$ \\
\hline 16 niveles & $15.00 \mathrm{~m}$ & 225 & $23.00 \mathrm{~m}$ \\
\hline
\end{tabular}

Es valioso hacer notar que la distribución de los pilotes de fricción diseñados es simétrica (figura 5), pero no es uniforme, puesto que para hacer una propuesta de diseño eficiente, se tomó en cuenta que los marcos contraventeados producen descargas verticales más grandes a la cimentación.

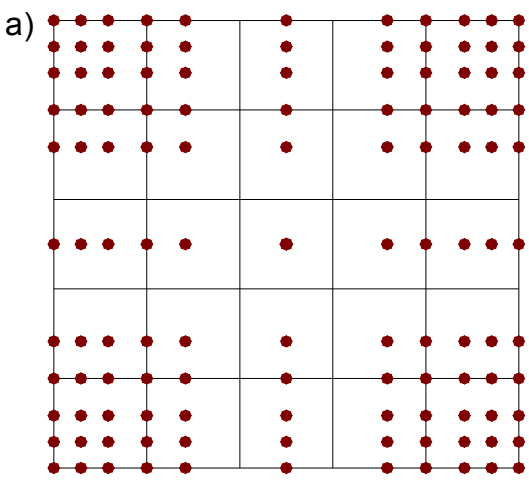

a) Edificio de 8 pisos

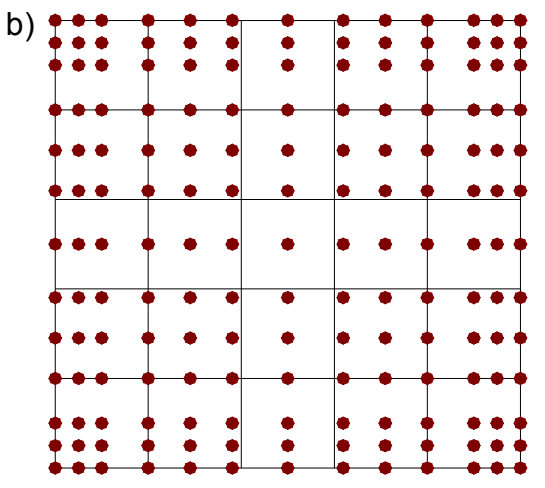

b) Edificio de 12 pisos

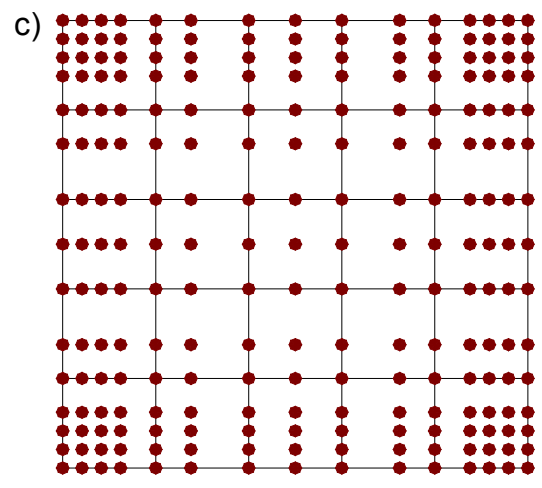

c) Edificio de 16 pisos

Figura 5. Esquema de la distribución de los pilotes de fricción en planta para los edificios en estudio

\section{CÁLCULO DE LAS FUNCIONES DE IMPEDANCIA}

En los análisis, las propiedades del sistema suelo - cimentación se consideraron a través de funciones de impedancia. Las funciones de impedancia son las rigideces dinámicas de la cimentación rígida sin masa, las cuales se definen como las fuerzas y momentos necesarios para producir desplazamientos y rotaciones armónicos unitarios en la cimentación. Las funciones son dependientes de la frecuencia de la excitación $\omega$ y se modelan como resortes y amortiguadores equivalentes a las propiedades mecánicas del suelo.

El análisis simplificado de la IDSE consiste en modelar el edificio sobre resortes y amortiguadores que representan la rigidez y el amortiguamiento del sistema suelo - cimentación. Con fines de diseño, los efectos de interacción suelo - estructura suelen tenerse en cuenta únicamente para el modo fundamental de vibración de la estructura. Una función de impedancia se expresa como se muestra en la ecuación 4 (Gazetas, 1991).

$$
K *(\omega)=K(\omega)+i \omega C(\omega)
$$

En la ecuación anterior, $K^{*}(\omega)$ es la función de impedancia del sistema suelo - cimentación, $K(\omega)$ es la rigidez dinámica del sistema suelo - cimentación, $C(\omega)$ es el coeficiente dinámico de amortiguamiento viscoso equivalente y $\omega$ es la frecuencia angular de la excitación. La rigidez dinámica $K(\omega)$ representa la 
rigidez e inercia del suelo en el que se encuentra la cimentación, donde la inercia es dependiente de la frecuencia de excitación, mientras que la parte imaginaria de la ecuación 4 expresa la energía disipada a través de su amortiguamiento $C(\omega)$.

Es común definir distintas magnitudes de las funciones de impedancia de acuerdo a la dirección en que se excita el sistema suelo - cimentación para representarlo como un juego de resortes y amortiguadores viscosos como se muestra en la figura 6 . En la figura, $K_{r}$ es la rigidez rotacional, $K_{h}$ es la rigidez horizontal y $K_{v}$ es la rigidez vertical que en este estudio se modeló como infinitamente rígida $\left(K_{v} \rightarrow \infty\right)$ ya que no se considera la influencia de la componente vertical del sismo. Además, debido a que las estructuras se estudiaron ante análisis no lineales estáticos, el amortiguamiento rotacional $C_{r}$, horizontal $C_{h}$ y vertical $C_{v}$ no se consideraron.

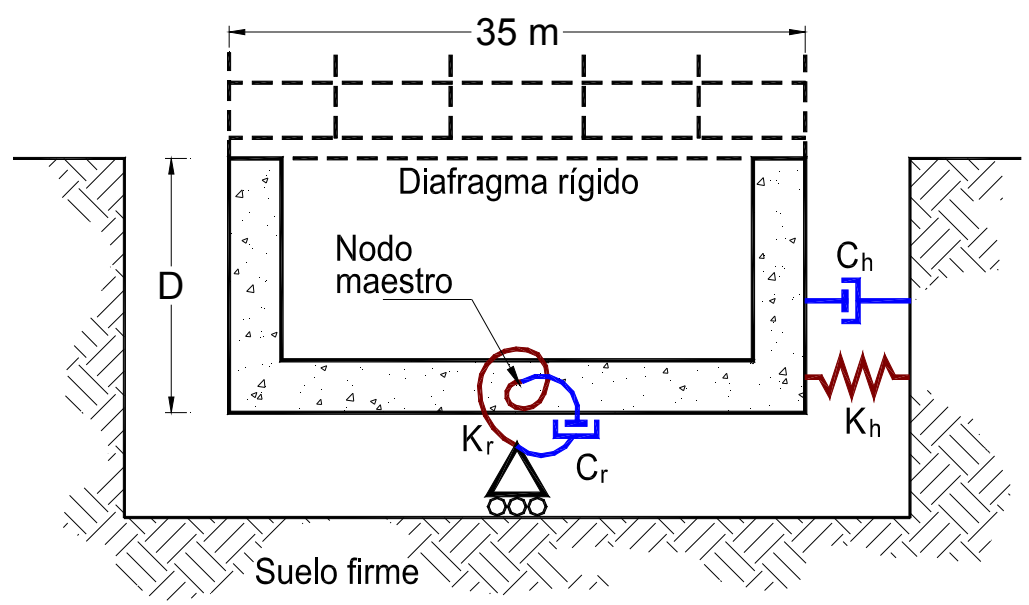

Figura 6. Resortes y amortiguadores equivalentes del suelo

En este estudio, las funciones de impedancia se determinaron mediante el programa Dyna5 (Novak et al., 1995), que permite calcular la respuesta de varios tipos de cimentaciones (rígidas o flexibles) ante una variedad de cargas dinámicas. El programa analiza las cimentaciones con diferentes profundidades de enterramiento y proporciona las funciones de impedancia para cualquier intervalo de frecuencias.

En el programa Dyna5 el cajón de cimentación se modeló como un esquema de stratum foundation, que considera el sistema como una cimentación superficial sobre un estrato homogéneo de espesor finito como se muestra en la figura 7 a. En la figura, $H_{s}$ es el espesor del estrato, $D$ es la profundidad de desplante y $Z c$ es la posición del centro de masa del cajón. Por otra parte, la cimentación con pilotes de fricción se utilizó el modelo pile foundation, que permite analizar grupos de pilotes desplantados en un medio estratificado, aunque en el presente estudio, debido a que el medio es homogéneo sólo se utilizó un estrato (figura $7 b$ ). Para el cálculo de la funciones de impedancia del grupo de pilotes se consideraron los efectos de grupo. 


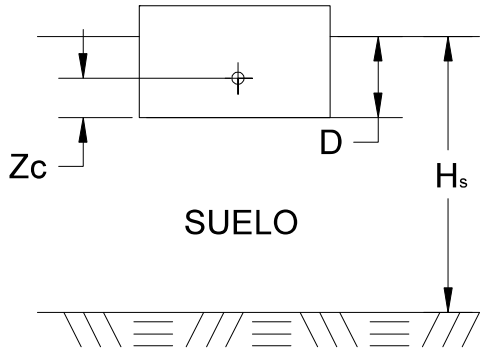

a) Modelo para el cajón

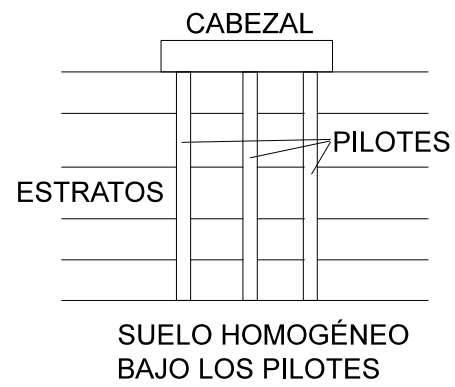

b) Modelo para el grupo de pilotes

Figura 7. Modelos matemáticos para las funciones de impedancia (adaptado de Novak et al., 1995)

En el programa empleado, los resultados del análisis de las cimentaciones se presentan en gráficas de la rigidez dinámica del sistema suelo-cimentación modelado y su variación con la frecuencia. El programa calcula la rigidez dinámica de traslación horizontal y vertical, rigidez de rotación, rigidez de torsión y una rigidez acoplada. En esta investigación, se tomaron en cuenta únicamente las componentes de traslación horizontal $K_{h}$ y de rotación $K_{r}$ para las frecuencias fundamentales obtenidas de los análisis sobre base rígida (tabla 2). Esta consideración supone que la mayor parte del movimiento estará asociada con el modo fundamental de vibrar de la estructura, como se propone en el planteamiento de las NTCS-04 MOCS08. Así, en la tabla 5 se reporta la magnitud de las funciones de impedancia para cada tipo de cimentación obtenidas de los análisis del Dyna5 (Novak et al., 1995).

Tabla 5. Funciones de impedancia para las frecuencias fundamentales según el tipo de cimentación

\begin{tabular}{lccccc}
\hline Modelo & $\begin{array}{c}\text { Frecuencia } \\
\text { fundamental de la } \\
\text { estructura con base } \\
\text { rígida }(\mathrm{rad} / \mathrm{s})\end{array}$ & $\begin{array}{c}\text { Rigidez } \\
\text { dinámica } \\
\text { horizontal, } K_{h} \\
(\mathrm{t} / \mathrm{cm})\end{array}$ & $\begin{array}{c}\text { Rigidez } \\
\text { dinámica } \\
\text { rotacional, } K_{r} \\
(\mathrm{t}-\mathrm{cm})\end{array}$ & $\begin{array}{c}\text { Cajón de cimentación } \\
\text { Rigidez } \\
\text { dinámica } \\
\text { horizontal, } K_{h} \\
(\mathrm{t} / \mathrm{cm})\end{array}$ & $\begin{array}{c}\text { Rigidez } \\
\text { dinámica } \\
\text { rotacional, } K_{r} \\
(\mathrm{t}-\mathrm{cm})\end{array}$ \\
\hline Edificio de 8 pisos & 15.71 & $1.203(10)^{3}$ & $7.472(10)^{9}$ & $1.043(10)^{3}$ & $9.104(10)^{8}$ \\
Edificio de 12 pisos & 12.57 & $9.533(10)^{2}$ & $3.394(10)^{9}$ & $1.451(10)^{3}$ & $2.846(10)^{8}$ \\
Edificio de 16 pisos & 9.97 & $9.053(10)^{2}$ & $3.903(10)^{9}$ & $2.036(10)^{3}$ & $5.644(10)^{8}$ \\
\hline
\end{tabular}

\section{ANÁLISIS NO LINEALES}

Se realizaron análisis no lineales ante carga estática monótona creciente con el programa SAP2000 v.15 (CSI 2011) ante un patrón de cargas laterales de forma triangular invertido siguiendo los criterios del método estático de las NTCS-04, que asume que el primer modo regirá la respuesta dinámica de las estructuras.

El programa utilizado realiza un modelado de la no linealidad de los elementos con base en la propuesta del FEMA 356 (FEMA 356, 2000) mediante curvas momento - rotación no lineal para vigas y columnas y/o carga - desplazamiento para contravientos como se muestra en la figura 8. La magnitud de los parámetros que definen la curva de capacidad de los elementos ( $a, b$ y c) se establecen para elementos de acero a partir de tablas que estipula el FEMA 356, que se obtuvieron mediante pruebas experimentales y análisis numéricos. Por otra parte, la carga crítica de pandeo por compresión $P_{c r}$ de los contravientos se 
definió dependiendo de la sección transversal por entrepiso para cada edificio como se discute en Tapia y Tena (2013).

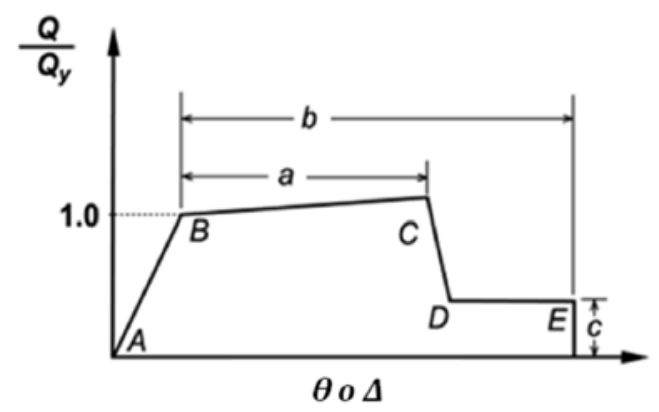

Figura 8. Momento-curvatura o fuerza-desplazamiento para elemento de acero según FEMA 356 (2000)

El comportamiento no lineal de la estructura se estudió considerando la respuesta inelástica de los elementos estructurales; esto es, considerando que las vigas y columnas pueden desarrollar articulaciones plásticas ubicadas al 5 y $95 \%$ de la longitud del elemento y que los contravientos se plastificarían por fluencia en tensión o pandeo lateral en compresión ubicando la falla al 50 por ciento de la longitud del contraviento.

\section{Modelo de la IDSE}

En los modelos desplantados sobre base flexible (cimentación con un cajón y con pilotes de fricción), la IDSE se representa por medio de un resorte rotacional de rigidez $K_{r}$ y un resorte traslacional horizontal de rigidez $K_{h}$ ubicados en un nodo maestro en el centro de la base, donde la base del edificio se modela con un diafragma infinitamente rígido (figura 6).

Debido a que en este estudio se analizarán marcos planos de los edificios estudiados y a que las funciones de impedancia (tabla 5) corresponden al sistema suelo-cimentación del edificio completo, fue necesario determinar la magnitud proporcional de la rigidez del sistema suelo - cimentación $\left(K_{r}\right.$ y $\left.K_{h}\right)$ que le corresponde a cada marco. Para ello, las constantes de los resortes que se emplearon en los análisis con base flexible se definieron considerando la proporción a la rigidez lateral total que aporta cada marco. Los porcentajes de rigidez lateral obtenidos de los tres tipos de marcos estudiados: marco sin contravientos, marco con una crujía contraventeada y marcos con dos crujías contraventeadas (figura 2) se muestran en la tabla 6. Es valioso hacer notar que la rigidez total del edificio (100\%) se obtiene de la suma del total de los seis marcos que conforman cada dirección global. Estos porcentajes se calcularon mediante las rigideces laterales de cada marco obtenida en los análisis y la rigidez lateral de todo el edificio.

Tabla 6. Porcentajes de rigidez lateral por marco

\begin{tabular}{lccc}
\hline \multicolumn{1}{c}{ Modelo } & $\begin{array}{c}\text { Marco sin } \\
\text { contravientos }\end{array}$ & $\begin{array}{c}\text { Marco con una crujía } \\
\text { contraventeada }\end{array}$ & $\begin{array}{c}\text { Marco con dos crujías } \\
\text { contraventeadas }\end{array}$ \\
\hline Edificio de 8 pisos & $5.42 \%$ & $16.64 \%$ & $27.94 \%$ \\
\hline Edificio de 12 pisos & $6.11 \%$ & $16.34 \%$ & $27.55 \%$ \\
\hline Edificio de 16 pisos & $7.15 \%$ & $16.43 \%$ & $26.42 \%$ \\
\hline
\end{tabular}


En la tabla 7 se muestran los periodos de vibrar de los marcos de acero considerados en este estudio. En ella, BR se refiere a los análisis que suponen un sistema suelo-cimentación infinitamente rígido (base rígida) y BF-Pilotes y BF-Cajón se refiere a los análisis con base flexible usando pilotes de fricción y el cajón de cimentación, respectivamente.

Tabla 7. Periodo fundamental de los marcos con base rígida (BR) y base flexible (BF)

\begin{tabular}{clccc}
\hline \multicolumn{1}{c}{ Modelo } & Marco & BR & BF-Pilotes & BF-Cajón \\
\hline \multirow{3}{*}{ Edificio de 8 pisos } & Sin contravientos & 1.19 & 1.38 & 1.86 \\
& Una crujía contraventeada & 0.68 & 0.79 & 1.07 \\
& Dos crujías contraventeadas & 0.53 & 0.61 & 0.83 \\
\hline \multirow{3}{*}{ Edificio de 12 pisos } & Sin contravientos & 1.56 & 2.03 & 2.08 \\
& Una crujía contraventeada & 0.87 & 1.18 & 1.21 \\
& Dos crujías contraventeadas & 0.68 & 0.92 & 0.95 \\
\hline \multirow{3}{*}{ Edificio de 16 pisos } & Sin contravientos & 1.81 & 2.44 & 2.22 \\
& Una crujía contraventeada & 1.12 & 1.32 & 1.42 \\
& Dos crujías contraventeadas & 0.88 & 1.26 & 1.15 \\
\hline
\end{tabular}

En todos los casos la IDSE incrementa el periodo fundamental de los marcos analizados con base rígida. Cuando el edificio es de baja altura (modelo de 8 pisos), la estructura más flexible se obtiene mediante el modelado de una cimentación con cajón de cimentación; sin embargo, en edificios altos (el modelo de 16 pisos), la estructura más flexible se obtiene considerando una cimentación con pilotes por fricción. Esta observación denota una ligera dependencia de la flexibilidad de los modelos entre la altura de los edificios y el sistema de cimentación, siendo que el modelo de 12 pisos es el caso intermedio.

\section{ANÁLISIS DE RESULTADOS}

En la figura 9 se muestran las curvas de capacidad globales de los marcos considerando el cortante basal contra la distorsión total (desplazamiento de la azotea entre la altura del edificio). En ellas, se muestra el desplazamiento total $\tilde{U}$ provocado por la deformación de la estructura $U$ más la deformación asociada a la rotación de la cimentación $\phi h$ y la deformación asociada a la de la cimentación $U_{C I M E N T}$ (figura 1). Estos resultados se utilizaron para compararlas contra las ecuaciones que evalúan la influencia de la flexibilidad de la cimentación en la ductilidad que pueden desarrollar las estructuras (NTCS-04; Avilés y Pérez-Rocha, 2005).

En las curvas de capacidad el último paso se definió como el incremento de carga cuando ocurría una de las siguientes condiciones:

a. Alguno de los elementos estructurales alcanzara la deformación última que puede desarrollar físicamente (rotación en trabes y columnas y/o alargamiento ó acortamiento en contravientos), definida como el punto $\mathrm{C}$ de la figura 8 en el programa de análisis empleado.

b. Se forme un mecanismo de piso débil que se definió como la articulación en ambos extremos de todas las columnas de un mismo entrepiso.

c. Se desarrolle un mecanismo plástico de nudo por la articulación de los extremos de todos los elementos que concurren en él.

En las curvas de capacidad se nota que a medida que el sistema suelo-cimentación es más flexible, la rigidez elástica del sistema es menor (figura 9). Asimismo, se aprecia una dependencia, que se había hecho notar anteriormente, entre la altura del modelo y el sistema de cimentación que es más flexible; es 
decir, el edificio de 8 pisos es más flexible cuando el modelo está resuelto con un cajón de cimentación; mientras que el edificio de 16 pisos está relacionado con mayores desplazamientos con una cimentación con pilotes de fricción. El edificio de 12 pisos tiene una respuesta de los modelos con cimentación flexible muy parecida.
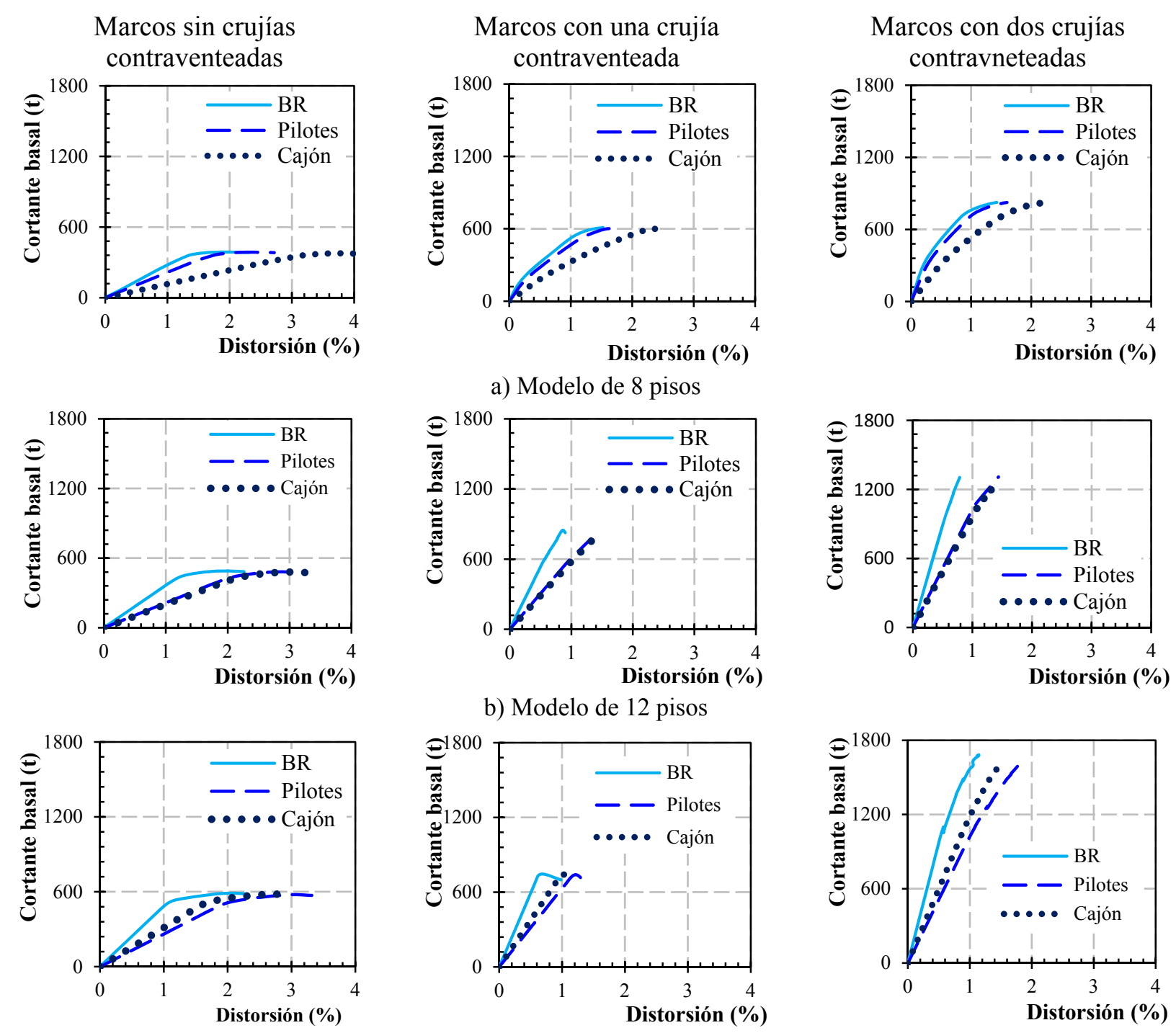

b) Modelo de 12 pisos
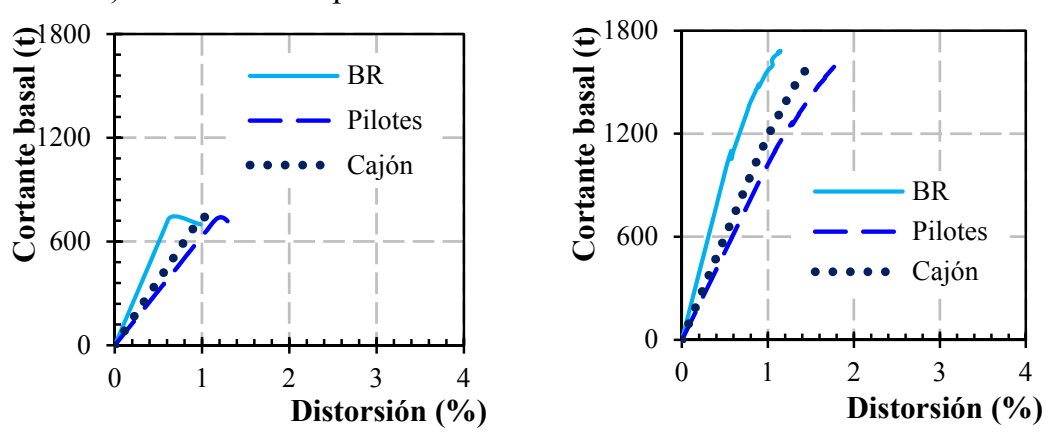

c) Modelo de 16 pisos

Figura 9. Curvas de capacidad de los marcos estudiados

Además, se calcularon curvas de capacidad que consideran únicamente la deformación de la estructura $U$ (figura 1) para comparar su magnitud con la obtenida de los análisis con base rígida, esto con la finalidad de estudiar si la flexibilidad de la base modifica el comportamiento asociado exclusivamente con la deformación de la estructura (figura 10). Considerando únicamente este desplazamiento, resultó que la deformación de la estructura que desarrollan los marcos es muy similar entre los análisis que consideran una base rígida y una base flexible. 


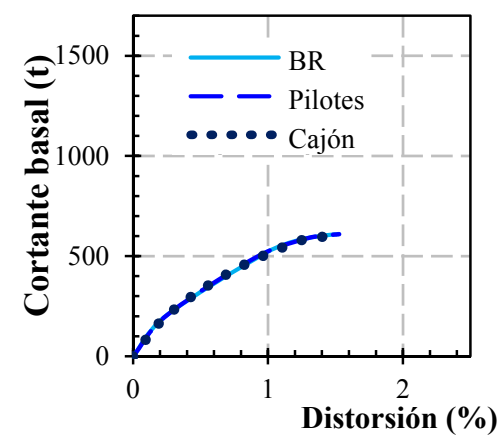

a) Marco de 8 pisos con una crujía contraventeada

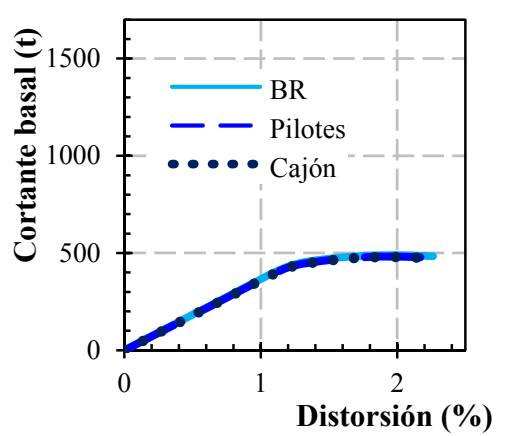

b) Marco de 12 pisos sin contravientos

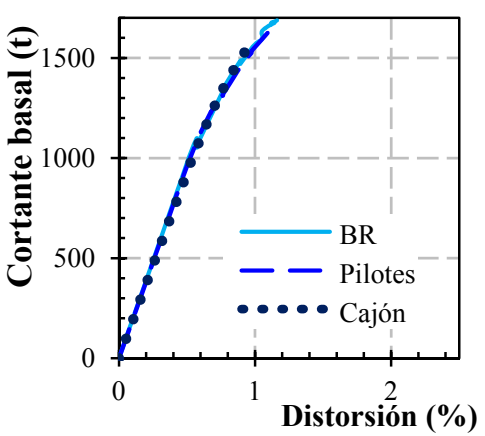

c) Marco de 16 pisos con dos crujías contraventeadas

Figura 10. Curvas de capacidad considerando únicamente la deformación de la estructura

Con el propósito de hacer más notoria la modificación de la respuesta inelástica asociada a la IDSE cuando se toma en cuenta únicamente la deformación de la estructura $U$, se calculó la respuesta inelástica para distintas magnitudes hipotéticas de la rigidez rotacional $K_{r}$. Así, en la figura 11 se muestran algunas de las curvas de capacidad calculadas en los análisis del marco sin contravientos y marco con dos crujías contraventeadas del edificio de 16 pisos. En ella, la curva de magnitud $K_{r}$ representa el comportamiento del análisis con la función de impedancia real, mientras que el resto de las curvas representan situaciones hipotéticas de suelos muy flexibles representados como una proporción de la función de impedancia.

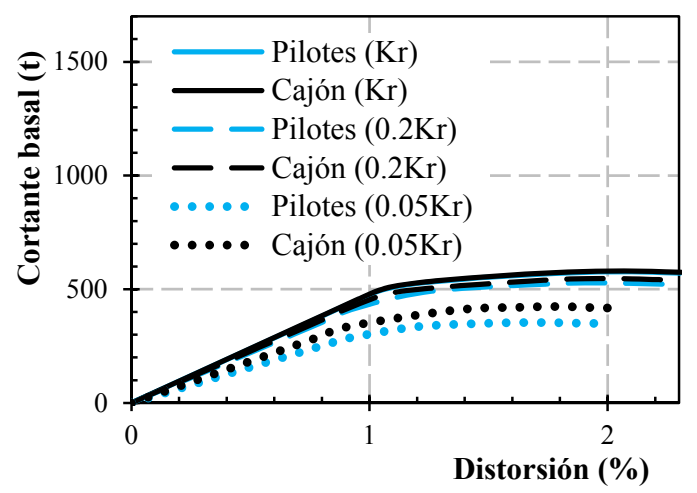

a) Marco sin contravientos

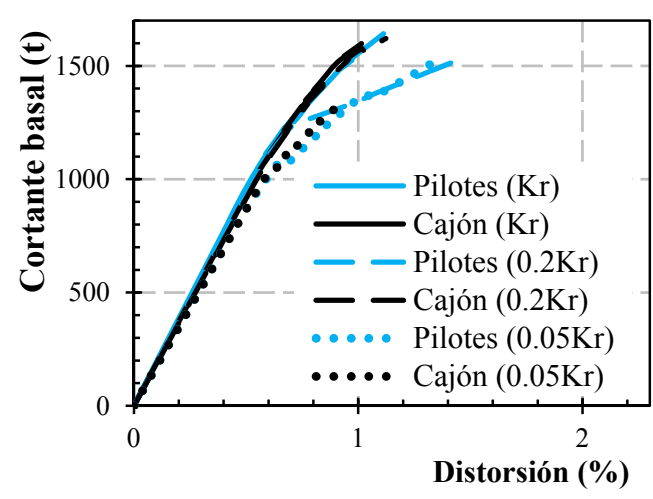

b) Marco con dos crujías contraventeadas

Figura 11. Curvas de capacidad del edificio de 16 pisos para distintas proporciones de $K_{r}$

En la figura 11, se nota que la cimentación con pilotes de fricción es la solución más flexible en el edificio de 16 pisos y esta tendencia, comentada anteriormente, parece ser independiente de la flexibilidad del suelo, aún hasta magnitudes hipotéticas de $K r$. En general, la IDSE afecta la resistencia, rigidez y capacidad de deformación que pueden desarrollar los modelos únicamente en situaciones hipotéticas, lo que implica que los efectos $P-\triangle$ asociados a la IDSE tienen poca relevancia, al menos para los marcos de esta investigación, cuando se consideran las propiedades de suelo reales incluyendo suelos con poca rigidez rotacional. 


\section{Ductilidades desarrolladas}

Debido a que hay diferencias más notorias entre los resultados de los marcos menos rígidos, la siguiente discusión se centra en los análisis de marcos sin contravientos y los marcos con una crujía contraventeada; sin embargo, es posible conocer los detalles de los resultados de los marcos con dos crujías contraventeadas en Dávalos (2013).

A partir de las curvas de capacidad se construyeron curvas primarias idealizadas (figura 12) que son las que usualmente consideran los reglamentos para definir el comportamiento inelástico del sistema. Las curvas bilineales se determinaron con el cortante de fluencia $V_{y}$, la distorsión de fluencia $\delta_{y}$ y el cortante máximo $V_{u}$. En ellas, la rigidez inicial $K_{\text {elast }}$ es la pendiente de la curva en el intervalo elástico, la rigidez post-fluencia se consideró con una pendiente igual a $k_{2}=0$ y la distorsión de fluencia teórica $\delta_{y}$ se definió como el punto de intersección entre ambas rectas.

En la tabla 8 se muestran las magnitudes de las ductilidades $\mu$ calculadas a partir de las curvas idealizadas bilineales de los análisis de los marcos de los modelos de 8, 12 y 16 pisos. Los cálculos se desarrollaron considerando las curvas de capacidad que consideran el desplazamiento total de los marcos (es decir, $\tilde{U}=U+\phi h+U_{C I M E N T}$ ) y las curvas que consideran únicamente la deformación de la estructura $U$. Los resultados hacen notar que la IDSE tiene influencia en la ductilidad $\mu$ que los marcos desarrollan debido a que se reduce la rigidez lateral del sistema y se modifica la capacidad de deformación. En la mayoría de los casos, los análisis de modelos con cimentaciones flexibles son menos dúctiles en comparación de los resultados de los análisis con base rígida.

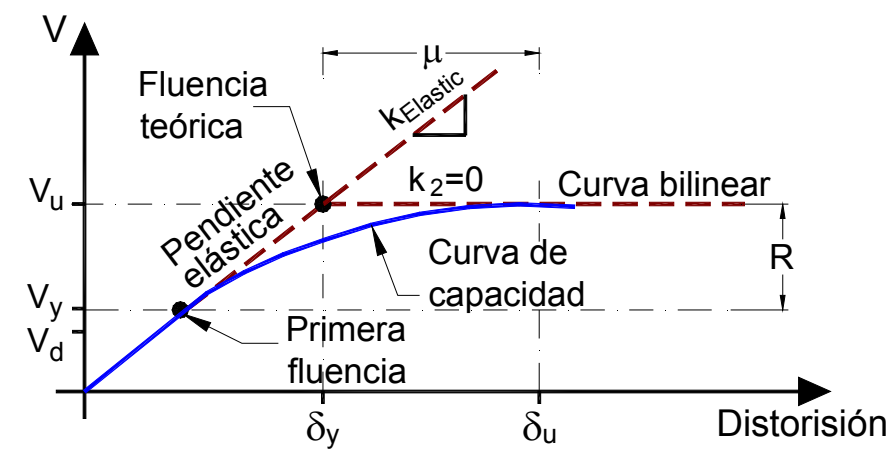

Figura 12. Definición de la curva bilineal idealizada

Igualmente, se nota que las ductilidades calculadas considerando únicamente el desplazamiento de la estructura $U$ son bastante similares a los resultados que se calcularon considerando base rígida. Esto implica que la reducción en la ductilidad calculada está asociada a la reducción en la rigidez elástica $k_{\text {Elastic }}$ (figura 1), previamente identificada en Pérez-Rocha y Avilés (2003). Sin embargo, es valioso hacer notar que los modelos de 12 y 16 niveles con una crujía contraventeada no siguen esa tendencia. Estos resultados coinciden con otras investigaciones (Ganjavi y Hao 2011; Tapia y Tena 2013) que sugieren una dependencia de la ductilidad que las estructuras pueden desarrollar en función de su altura. De hecho, esta dependencia entre la ductilidad y la altura de los edificios ya se ha incluido en el reglamento de Canadá para el diseño sísmico de estructuras metálicas (Izvernari et al., 2007). 
Tabla 8. Ductilidades teóricas desarrolladas en los análisis de los marcos estudiados

\begin{tabular}{|c|c|c|c|c|c|c|}
\hline \multirow{3}{*}{ Modelo } & \multirow{3}{*}{ Edificio } & \multicolumn{5}{|c|}{ Ductilidades desarrolladas } \\
\hline & & \multirow{2}{*}{$\begin{array}{l}\text { Base } \\
\text { rígida, } \\
\text { BR }\end{array}$} & \multicolumn{2}{|c|}{$\begin{array}{l}\text { Curva de capacidad con el } \\
\text { desplazamiento total, } \tilde{U}\end{array}$} & \multicolumn{2}{|c|}{$\begin{array}{c}\text { Curva de capacidad con la } \\
\text { deformación de la estructura, } U\end{array}$} \\
\hline & & & Cajón & Pilotes & Cajón & Pilotes \\
\hline \multirow{3}{*}{$\begin{array}{l}\text { Marcos sin } \\
\text { contravientos }\end{array}$} & 8 pisos & 1.66 & 1.29 & 1.51 & 1.66 & 1.66 \\
\hline & 12 pisos & 1.69 & 1.39 & 1.41 & 1.69 & 1.69 \\
\hline & 16 pisos & 1.89 & 1.60 & 1.51 & 1.89 & 1.89 \\
\hline \multirow{3}{*}{$\begin{array}{l}\text { Marcos con una } \\
\text { crujía } \\
\text { contraventeada }\end{array}$} & 8 pisos & 2.05 & 1.47 & 1.82 & 2.05 & 2.05 \\
\hline & 12 pisos & 1.14 & 1.03 & 1.05 & 1.05 & 1.08 \\
\hline & 16 pisos & 1.56 & 1.16 & 1.18 & 1.41 & 1.31 \\
\hline
\end{tabular}

Asimismo, es posible concluir que las distorsiones obtenidas de análisis con base rígida son una buena representación de las distorsiones que se obtendrían en análisis con base flexible cuando se considera la deformación de la estructura de la estructura $U$ únicamente. Pese a esto, cabe mencionar que el modelo con base rígida subestima el desplazamiento total que podría desarrollar una estructura con una cimentación flexible.

Ahora, las NTCS-04 proponen un criterio para estimar la ductilidad que desarrollará una estructura, a partir de su respuesta con base rígida mediante la aplicación de la ecuación 1. En la tabla 9 se muestran las ductilidades estimadas para estructuras con base flexible considerando este procedimiento. En los cálculos se tomaron en cuenta los periodos fundamentales de la estructura con base rígida $T_{e}$ y los obtenidos para los modelos con base flexible mostrados en la tabla 7 y las ductilidades con base rígida $\mu$ mostradas en la tabla 8. En la tabla 9, ${ }_{p N T C} \mathrm{y}_{c N T C}$ indica la ductilidad estimada mediante el criterio de las NTCS-04 para el caso de una cimentación con pilotes por fricción y con un cajón de cimentación, respectivamente. En el cálculo sólo se utilizó la expresión para la modificación de la ductilidad incluida en las NTCS-04 (ecuación 1). La determinación de las magnitudes del periodo con base rígida $\left(T_{e}\right)$ y con base flexible $\left(\widetilde{T}_{e}\right)$ se realizó mediante los modelos numéricos considerando las rigideces dinámicas calculadas con el programa Dyna5. Estos cálculos se comparan contra la ductilidad estimada en las curvas de capacidad que consideran únicamente la deformación de la estructura $U$ de la tabla 8.

Tabla 9. Evaluación del criterio de estimación de la ductilidad con base flexible según NTCS-04

\begin{tabular}{ccccccccc}
\hline \multirow{2}{*}{$\begin{array}{c}\text { Modelo } \\
\text { Edificio }\end{array}$} & \multicolumn{2}{c}{$\begin{array}{c}\text { Ductilidades obtenidas } \\
\text { en los análisis }\end{array}$} & $\begin{array}{c}\text { Ductilidades } \\
\text { estimadas según } \\
\text { NTCS-04 }\end{array}$ & \multicolumn{2}{c}{$\begin{array}{c}\text { Porcentajes de } \\
\text { variación }\end{array}$} \\
\cline { 2 - 9 } & & $\mu$ & $\mu_{p}$ & $\mu_{c}$ & $p N T C$ & $c N T C$ & $p N T C / \mu_{p}$ & $c N T C / \mu_{c}$ \\
\hline \multirow{2}{*}{$\begin{array}{c}\text { Marco sin } \\
\text { contravientos }\end{array}$} & 8 pisos & 1.66 & 1.51 & 1.29 & 1.49 & 1.27 & 0.99 & 0.98 \\
\cline { 2 - 9 } & 12 pisos & 1.69 & 1.41 & 1.39 & 1.40 & 1.39 & 1.00 & 1.00 \\
\hline \multirow{2}{*}{$\begin{array}{c}\text { Marco con una } \\
\text { crujía } \\
\text { contraventeada }\end{array}$} & 16 pisos & 1.89 & 1.51 & 1.60 & 1.49 & 1.59 & 0.99 & 0.99 \\
\cline { 2 - 9 } & 12 pisos & 1.14 & 1.05 & 1.03 & 1.08 & 1.07 & 1.02 & 1.04 \\
\hline
\end{tabular}


Con base en los resultados, el criterio de estimación de las NTCS resulta ser una buena aproximación de la ductilidad que desarrollan los marcos sin contravientos. Sin embargo, ante el aumento de rigidez lateral (marcos con una crujía contraventeada), los resultados indican una tendencia desfavorable, que parece ser dependiente de la altura de los modelos.

Aunado a lo anterior, las NTCS-04 establecen un criterio para determinar la importancia de la influencia de la IDSE en el comportamiento de la estructura, a partir de la rigidez relativa de las estructuras respecto a la rigidez del sistema suelo - cimentación (ecuación 5). Según este planteamiento, los efectos de la IDSE son relevantes y no deberían de despreciarse cuando la magnitud del cociente es menor a $\lambda<2.5$.

$\lambda=\frac{T_{e} H_{s}}{T_{s} h}$

En la ecuación anterior, $H_{s}$ es el espesor del estrato, $h$ es la altura de la estructura para el caso de estructuras de un solo grado de libertad (figura 1). Para el caso de estructuras de varios grados de libertad, la altura $h$ corresponde a la altura efectiva en el primer modo. $T_{s}$ y $T_{e}$ son el periodo dominante del estrato de suelo y de la estructura, respectivamente. En la tabla 10 se muestran los cálculos obtenidos para evaluar la importancia de los efectos de la interacción suelo - estructura en los edificios estudiados en esta investigación. En los cálculos el periodo del suelo se consideró igual a $T_{s}=1.85 \mathrm{~s}$ (tabla 3 ); el espesor del estrato igual a $H_{s}=40 \mathrm{~m}$ considerando el estudio de Mecánica de Suelos; los periodos $T_{e}$ de los edificios mostrados en la tabla 2 y siguiendo el planeamiento de las NTCS la altura efectiva de la estructura se consideró igual a $h=0.7 H_{T}$, donde $H_{T}$ es la altura total del edificio de la tabla 2.

Tabla 10. Importancia de la IDSE según NTCS-04

\begin{tabular}{lcc}
\hline \multicolumn{1}{c}{ Modelo } & $h=0.7 H_{T}$ & $\lambda$ \\
\hline Edificio de 8 pisos & $19.6 \mathrm{~m}$ & 0.441 \\
\hline Edificio de 12 pisos & $29.4 \mathrm{~m}$ & 0.368 \\
\hline Edificio de 16 pisos & $39.2 \mathrm{~m}$ & 0.348 \\
\hline
\end{tabular}

Con base en los resultados, los efectos de la IDSE son relevantes en los edificios estudiados localizados en suelos con poca rigidez rotacional (suelo blando). Asimismo, se nota nuevamente que los efectos son dependientes de la altura de los edificios.

\section{CONCLUSIONES}

El artículo presenta los resultados de análisis no lineales ante carga estática monótona creciente de marcos de acero de edificios regulares para estudiar la influencia de la interacción dinámica suelo estructura (IDSE) en su comportamiento inelástico. En el estudio se eligieron marcos sin contravientos, con una crujía contraventeada y con dos crujías contraventeadas para considerar la influencia de la rigidez lateral en los resultados.

Los edificios estudiados, que tienen 8,12 y 16 pisos, se supusieron localizados en la zona IIIb para asociarlo a las mayores demandas de aceleración que proponen las Normas Técnicas (NTCS-04) y fueron diseñados siguiendo estrictamente los criterios del Reglamentos de Construcciones para el Distrito Federal (RCDF-04). A fin de estimar los efectos de la IDSE en la respuesta inelástica se consideraron tres casos: a) 
análisis de los edificios considerando un sistema suelo-cimentación infinitamente rígido (base rígida), b) análisis con una cimentación con pilotes de fricción y c) análisis con un cajón de cimentación.

El diseño de la cimentación se realizó mediante los criterios de las Normas Técnicas (NTCC-04) y usando las propiedades estratigráficas de un estudio de la Colonia Roma, Delegación Cuauhtémoc en congruencia con las suposiciones del diseño elástico de los edificios. El estudio de los análisis con cimentación flexible se realizó desde dos perspectivas, considerando: a) únicamente el desplazamiento relacionado con la deformación de la estructura $U \mathrm{y}$ b) el desplazamiento total $\left(\tilde{U}=U+\phi h+U_{\text {CIMENT }}\right.$; es decir, considerando el desplazamiento relacionado con la deformación de la estructura $U$ más el giro de la cimentación $\phi h$ más el desplazamiento de la cimentación $U_{C I M E N T}$.

En los resultados obtenidos, se nota una ligera dependencia de la altura de los modelos $H_{e}$ y de la rigidez de los marcos en la reducción de la capacidad de deformación inelástica asociada a la IDSE. Con base en los resultados, se nota que las ductilidades calculadas considerando únicamente el desplazamiento de la estructura $U$ son bastante similares a los resultados que se calcularon considerando base rígida cuando se consideran marcos de acero sin contravientos; sin embargo, en edificios altos con marcos con contravientos no se sigue esa tendencia. Esta observación tiene relación con los resultados de otras investigaciones que señala que la ductilidad que desarrollan las estructuras es dependiente de la altura de los edificios.

El efecto de la IDSE modifica la ductilidad teórica que pueden desarrollar los edificios, dado que en la mayoría de los casos, los modelos con cimentaciones flexibles reportan una disminución de la ductilidad que puede desarrollar el sistema en comparación de los resultados con base rígida cuando se considera el desplazamiento total de los edificios $\left(\tilde{U}=U+\phi h+U_{C I M E N T}\right)$. Sin embargo, las distorsiones obtenidas de análisis con base rígida son una buena representación de las distorsiones que se obtendrían en análisis con base flexible cuando se considera la deformación de la estructura de la estructura $U$ únicamente. Pese a esto, se demostró que el modelo con base rígida subestima el desplazamiento total que podría desarrollar una estructura con una cimentación flexible.

Los resultados de los análisis inelásticos se compararon con la estimación de la ductilidad que desarrollará una estructura según las NTCS-04. Los resultados indican que el criterio de las NTCS-04 es una buena aproximación de la ductilidad que desarrollan los marcos de acero sin contravientos. Sin embargo, ante el aumento de rigidez lateral (marcos con contravientos) los resultados indican una tendencia desfavorable, que parece ser dependiente de la altura de los modelos.

Los resultados del estudio muestran que los efectos de la IDSE pueden ser relevantes en edificios altos estructurados con marcos de acero localizados en suelo blando dada la modificación de su desempeño inelástico. Este estudio representa una referencia racional de la variación de la rigidez inducida por la flexibilidad de la cimentación y constituyen un congruente indicio de la respuesta inelástica de la edificación en función de la IDSE; sin embargo, será necesario realizar otros estudios con una mayor cantidad de modelos antes de realizar conclusiones definitivas.

\section{AGRADECIMIENTOS}

Los autores agradecen la beca otorgada al tercer autor por el Consejo Nacional de Ciencia y Tecnología de México (CONACYT) para sus estudios de Maestría. Asimismo, agradecen al Dr. Alonso Gómez Bernal su apoyo con el estudio de mecánica de suelos del Valle de México. 


\section{REFERENCIAS}

Aguilar R H, M Galicia, M Salazar y J Avilés (2003), “Alteraciones de la respuesta dinámica del suelo debidas al hundimiento regional", Memorias, XVI Congreso Nacional de Ingeniería Sísmica, Ixtapa, Gro. Noviembre.

Avilés J y L E Pérez-Rocha (2005), "Soil-structure interaction in yielding systems", Earthquake Engineering and Structural Dynamics, Vol. 32, pp. 1749-1771.

Barcena A y L Esteva (2006) "Influence of dynamic soil-structure interaction on the nonlinear response and seismic reliability of multistory systems", Earthquake Engineering and Structural Dynamics, Vol. 36, pp. 327-346.

CSI (2011), "SAP2000 v. 15 Integrated Solution for Structural Analysis and Design". Computers and Structures, Inc. Berkeley, California. Estados Unidos.

Dávalos D (2013), "Influencia de la interacción dinámica suelo estructura en el comportamiento estático no lineal de marcos de acero con y sin contravientos", Tesis de Maestría, Posgrado en Ingeniería Estructural, Universidad Autónoma Metropolitana, México.

ESVM (1988), "Estudios sobre sismicidad en el valle de México", Secretaría General de Obras Públicas, Primera edición 1988, Departamento del Distrito Federal, DDF.

FEMA 356 (2000), "Handbook for the Seismic Evaluation of Buildings-A Prestandard, American Society of Civil Engineers". Federal Emergency Management Agency (FEMA), Washington, D.C.

Ganjavi B y H Hao (2011), "Elastic and inelastic response of single- and multi-degree-of-freedom systems considering soil structure interaction effects", Proceedings, Australian Earthquake Engineering Society, Australia.

Gazetas G (1991), Foundation vibrations, Foundation Engineering Handbook, Ed. H Y Fang, Van Nostrand Reinhold, Nueva York.

Ghannad M A y H Jahankhah (2007) "Site-dependent strength reduction factors for soil-structure systems", Soil Dynamics and Earthquake Engineering, Vol. 27, pp. 99-110.

Halabian A y S Kabiri (2004) "Soil structure interaction effects on inelastic response of R/C stack-like structures", Proceedings, 13th World Conference on Earthquake Engineering, Canada.

Izvernari C, M Lacerte y R Tremblay (2007), "Seismic performance of multi-storey concentrically braced steel frames designed according to the 2005 Canadian Seismic Provisions", Proceedings, $9^{\text {th }}$ Canadian Conference on Earthquake Engineering, Ottawa, Ontario, Canada. Junio.

MOCS-08 (2008), "Manual de Obras Civiles. Capítulo de Diseño por Sismo", Comisión Federal de Electricidad. Instituto de Investigaciones Eléctricas.

Mahsuli M y M A Ghannad (2009) "The effect of foundation embedment on inelastic response of structures", Earthquake Engineering and Structural Dynamics, Vol. 38, pp. 423-437 
NTCC-04 (2004), “Normas Técnicas Complementarias para Diseño de Cimentaciones”, Reglamento de Construcciones para el Distrito Federal, Gaceta Oficial del Departamento del Distrito Federal.

NTCS-04 (2004), "Normas Técnicas Complementarias para Diseño por Sismo", Reglamento de Construcciones para el Distrito Federal, Gaceta Oficial del Departamento del Distrito Federal.

Novak M, M Sheta, L El-Hifnawy, H El-Marsafawi y O Ramadan (1995), "DYNA5: A computer program for calculation of foundation response to dynamic loads", Geotechnical Research Centre, The University of Western Ontario, Canada.

Pérez-Rocha L E y J Avilés (2003), "Evaluación de efectos de interacción en resistencias inelásticas", Revista de Ingeniería Sísmica, No. 69, pp. 45-71.

Prakash V, G H Powell y F Fillipou (1992), "DRAIN-2DX: base program user guide", Report No. $U C B / S E M M-92 / 29$, Department of Civil Engineering, University of California at Berkeley.

RCDF-04 (2004), "Reglamento de Construcciones para el Distrito Federal", Gaceta Oficial del Departamento del Distrito Federal, Enero.

Rosenblueth E y D Reséndiz (1988), "Disposiciones reglamentarias de 1987 para tener en cuenta interacción dinámica suelo-estructura", Series del Instituto de Ingeniería, No. 509, Universidad Nacional Autónoma de México."

Tapia E y A Tena (2008), "Behavior of regular steel moment resisting concentrically braced frames (MRCDBFs) in seismic zones". Proceedings, XIV World Conference on Earthquake Engineering, Beijing, China, No. 05-05-0008. Octubre.

Tapia E y A Tena (2011), "Factores de ductilidad y sobrerresistencia en marcos de acero con contraventeo chevrón", Revista de Ingeniería Sísmica. No. 84 pp. 46-68. México, Distrito Federal. ISSN 0185092X.

Tapia E y A Tena (2013), "Diseño sísmico de marcos de acero contraventeados. Parte 1: Recomendaciones de diseño", Revista de Ingeniería Sísmica, SMIS. No.88. pp. 43-68. ISSN-0185-092X.

Wolf J P (1985), “Dynamic Soil-Structure Interaction”, Prentice-Hall, Nueva Jersey, Estados Unidos. 